\title{
MARCO CONCEPTUAL PARA UNA FORMACIÓN VIRTUAL DISTRIBUIDA INTEGRAL PARA LA VIDA A ESCALA MUNDIAL DESDE UN ENFOQUE ECOSISTÉMICO
}

\author{
Conceptual Framework for Distributed Virtual Training, Integral to Life on a Global Scale \\ from an Ecosystem Approach
}

Víctor GERMÁN SÁNCHEZ ARIAS

Universidad Abierta y Educación a Distancia (CUAED), México

Universidad Nacional Autónoma de México (UNAM), México

\section{KEY WORDS}

Educational ecosystem Virtuality

Information Societies

Integral education

Education for life

\section{ABSTRACT}

Thanks to information and communications technologies (ICT), education systems have had an unprecedented momentum, have been extended worldwide and can be accessible regardless of geographic location; But this potential could give it a greater boost, a system of distributed training, integrating formal, non-formal and informal education, with the world of work and culture. In this work, a theoreticalconceptual critical approach was carried out to support, from an ecosystem approach, the redesign of distributed and collaborative educational ecosystems for a distributed, comprehensive virtual education and for life on a world scale.

\section{PALABRAS CLAVE}

Ecosistemas educativos

Virtualidad

Sociedades de la información

Educación integral

Educación para la vida

\section{RESUMEN}

Gracias a las tecnologías de la información y las comunicaciones (TIC) los sistemas educativos han tenido un impulso sin precedente, se han extendido a escala mundial y pueden ser accesibles sin importar la localidad geográfica; pero este potencial podría darle un mayor impulso, un sistema de formación distribuida, integrando la educación formal, no formal e informal, con el mundo del trabajo y el cultural. En este trabajo se realizó un acercamiento crítico teórico-conceptual para fundamentar, desde un enfoque ecosistémico, el rediseño de ecosistemas educativos distribuidos y colaborativos para una formación virtual distribuida, integral y para la vida a escala mundial.

Recibido: $13 / 11 / 2019$

Aceptado: 20/02/2020

\section{GLOBAL KNOWHEDGE}




\section{Problemática: una formación virtual distribuida, integral y para la vida a escala mundial}

no de los fines de los sistemas de $\mathrm{U}$ educación formal es el de insertar a sus egresados al mundo social y del trabajo. Gracias a las TIC la educación se ha extendido, ya sea en las modalidades en línea o a distancia, incluso a escala mundial, rompiendo barreras geográficas y temporales; ahora se puede tener acceso a una formación desde cualquier parte del mundo y en cualquier momento sin necesidad de desplazarse físicamente. En este sentido, el uso de las TIC ha contribuido a resolver uno de los grandes problemas sociales del mundo, el derecho a la educación para la gran mayoría de la población. Una gran demanda que no podían resolver los sistemas tradicionales localizados geográficamente por la falta de espacios físicos y porque su modalidad requiere de la presencialidad de estudiantes y profesores en horarios específicos. En general en esta extensión se ha conservado, en el diseño y su operación, la estructura organizacional de la modalidad presencial que está regida por un control centralizado que define cada institución sobre su normatividad, su curriculum, su gestión y sus procesos de enseñanza y aprendizaje. Sin embargo, las TIC no solamente son extensiones, son toda una estructura organizada en la red Internet-WEB a escala mundial que permite la interconexión de sistemas autónomos abiertos que pueden ser de cualquier tipo, como los educacionales -formales, no formales e informales-, gubernamentales, empresariales, sociales, de entretenimiento, etc., de hecho esta red es la base del mundo virtualizado (una imagen del real) de las sociedades de la información, los conocimientos y el aprendizaje que está conformado por una red dinámica de sistemas socio virtuales altamente interconectados y distribuidos en la red y que finalmente ha dado paso a una nueva cultura de alcance mundial, la digital.

Si se considerará a todo este mundo virtual, premisa de la que parte esta propuesta, como el gran entorno de formación a escala mundial, cabe preguntarse, si la educación no podría dar, además del salto cuantitativo en su extensión, a otro cualitativo, con una modalidad de educación ampliada, distribuida, integral y para la vida a escala mundial. Para logar este salto, es necesario un nuevo enfoque organizacional en el rediseño de los sistemas educativos que transite de una estructura centralizada a una distribuida en red, que es la base con la que está organizado el mundo virtual. En resumen, el reto de este trabajo consiste en pasar del paradigma de sistemas educativos virtualizados y centralizados para el mundo, al de una red de ecosistemas educativos insertos en el mundo, donde conviven, colaboran y evolucionan con todos los otros sistemas que lo habitan con el propósito de ofrecer una formación virtual, distribuida, integral y para la vida.

Sin embargo, antes de plantear la propuesta, es importante comprender la naturaleza del mundo virtualizado y las bases del enfoque ecosistémico.

\section{Aproximación teórico-conceptual}

Este trabajo partió de las reflexiones de un proyecto de investigación interdisciplinaria, que está en proceso, y tiene como objetivo aplicar principios ecosistémicos a un sistema de educación formal a distancia ${ }^{1}$, que partió de la premisa sobre el gran potencial que tiene para la educación el considerar al mundo virtual de las sociedades de la información, los conocimientos y el aprendizaje como el gran entorno para una formación distribuida, integral y para la vida a escala mundial. Este potencial se debe a que en este gran espacio socio-virtual se encuentran:

a) insertos y distribuidos en el mundo una gran diversidad de sistemas educativos, del trabajo, de la cultura, etc.

b) el mayor repositorio de recursos, servicios, información, conocimientos, etc. que han sido producidos por los mismos sistemas y que están disponibles a través de navegadores WEB.

\footnotetext{
1 Proyecto de investigación Proyecto Ecosistemas Educativos, de la Coordinación de Universidad Abierta y Educación a Distancia (CUAED), de la Universidad Nacional Autónoma de México (UNAM), que tiene como objetivo la aplicación de principios ecosistémicos para el rediseño del Sistema de Universidad Abierta y Educación a $\begin{array}{lll}\text { distancia } & \text { SUAyED UNAM } & \end{array}$ https://ecoseduca.cuaed.unam.mx/).
} 
Este potencial es enorme, sin embargo, antes de plantear nuevos modelos educativos, se tienen que tomar en cuenta las diferentes estructuras en las que se sustentaría:

a) el soporte tecnológico que está organizado en una red dinámica y compleja de sistemas computacionales autónomos e interdependientes, que aparecen $y$ desaparecen en procesos continuos de adaptación y evolución

b) la virtualización distribuida de espacios sociales que tiene sus propias dinámicas y complejidades y que igualmente aparecen y desaparecen en procesos continuos de adaptación y evolución; y

c) el mundo virtual, deslocalizado, una imagen extendida del mundo real complejo, el cual está conformado por organizaciones, sociedades y culturas, localizados geográficamente, con sus propias aspiraciones $y$ contradicciones $y$ que comparten e impactan el medio ambiente del planeta.

Por todos estos factores, el rediseño de una formación en estos contextos cambiantes debe realizarse desde un nuevo enfoque organizativo que permita encontrar equilibrios en sus procesos dinámicos.

\subsection{El enfoque ecosistémico}

Para poder coordinar una red distribuida de sistemas autónomos y colaborativos es necesario plantear un nuevo paradigma organizacional flexible que vaya más allá de los sistemas planificados, centralizados y aislados; se requiere de mecanismos flexibles que permitan la coordinación, mediante acuerdos. En este trabajo se propone la metáfora ecosistémica como categoría organizativa.

Básicamente, desde la biología un ecosistema, según la Secretariat of the Convention on Biological Diversity (2004), se define como un entorno natural donde conviven seres bióticos de diversas especies que están en evolución, adaptándose continuamente a circunstancias no previstas para encontrar sus equilibrios en la producción y consumo de recursos abióticos mediante la colaboración, la competencia, la autorregulación y la autoorganización.
Un ecosistema social es un recorte donde conviven personas y comunidades que comparten servicios y recursos sociales que de acuerdo con la "Guía básica de conocimiento sobre medio ambiente", de Garrido (2006), éste está compuesto por cuatro variables que interaccionan entre sí: la organización social, la tecnología, la población y el medio ambiente.

A partir de estos contextos naturales y sociales, un ecosistema virtual educativo autónomo podría rediseñarse como un espacio flexible y colaborativo donde conviven, como seres bióticos, los estudiantes, profesores, comunidades, administradores y agentes computacionales (algoritmos y robots, programados con inteligencia artificial), que construyen, usan y gestionan continuamente recursos y servicios educativos abiertos (recursos abióticos) y están continuamente adaptándose por un lado, a sus procesos de gestión y de enseñanza-aprendizaje; y por otro, a las influencias de otros ecosistemas autónomos con los que conviven, como el económico, el social, el cultural, etc.

\subsection{Propuesta de un modelo de formación distribuida desde un enfoque ecosistémico virtual}

La propuesta de rediseño de ecosistemas educativos para una formación virtual, distribuida, integral y para la vida, se basa en considerar como el entorno de formación, al mundo virtual de las sociedades de la información, los conocimientos y el aprendizaje. Dicho entorno estaría conformado por redes de ecosistemas sociales de todo tipo, donde los educativos se rediseñarían como un espacio virtual organizado y gestionado dinámicamente por comunidades internas y externas de gestores, profesores, estudiantes y agentes computacionales con el fin de distribuir, usando la red, la formación, la gestión y los procesos de enseñanza y aprendizaje mediante mecanismos de autorregulación, autoorganización y negociación, contando con una libre circulación de información, conocimiento, experiencias, programas, procesos, recursos y de cualquiera de los actores educativos.

Mediante este rediseño, sería posible una formación que pueda adaptarse a los cambios, 
educativos, sociales y tecnológicos, internos y externos, para formarse no solamente navegando, tomando diferentes rutas, entre los diferentes espacios educativos de la red sino en todos los otros del ámbito social con los que se interactúa; que finalmente son los espacios donde se aprende durante la vida. Esta propuesta se representa en la figura1.

Figura 1. Modelo para una formación virtual distribuida, integral y para la vida desde un enfoque ecosistémico.
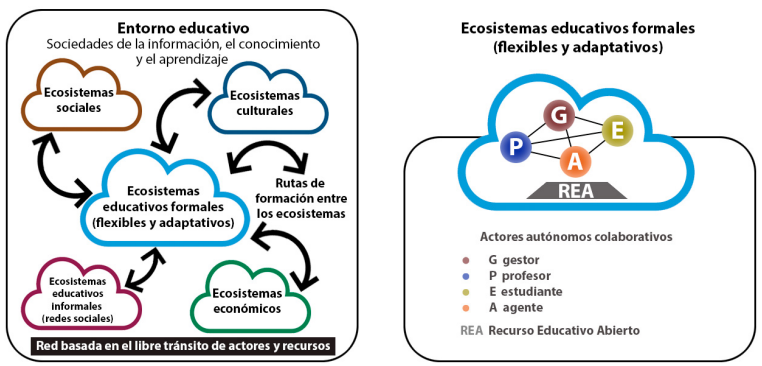

Fuente: elaboración propia, 2019.

\subsection{Marco conceptual de referencia}

Para la implantación de este modelo de formación, es necesario definir un marco flexible conceptual de referencia, que permita el rediseño de los ecosistemas educativos insertos en el gran entorno educativo que ofrece el mundo virtual de las sociedades de la información, los conocimientos y el aprendizaje, y que a su vez, está inserto e interactuando con el mundo real. Propuesta que se representa en la figura 2 .

Figura 2. Marco flexible conceptual de referencia.
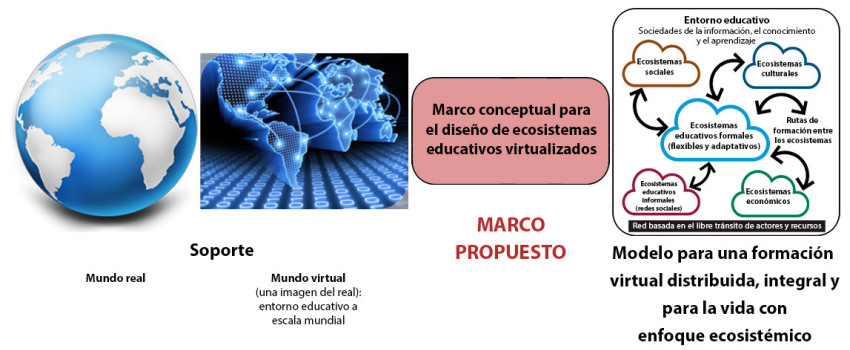

Fuente: elaboración propia, 2019

A pesar de que el concepto de ecosistema es un tema de actualidad y que ha sido abordado desde diferentes ámbitos, no existe aún un marco teórico-conceptual integral que le dé un sustento a un conjunto de principios flexibles de rediseño de ecosistemas educativos en el contexto interdisciplinario y complejo de formación como se planteó en este trabajo. Aprovechando las reflexiones de un proyecto previo de investigación interdisciplinaria ${ }^{2}$.

En este trabajo se presenta una primera aproximación de un marco que se realizó a partir de una investigación crítica exploratoria teóricaconceptual, que considera a la tecnología como un producto social, a partir de un análisis de las siguientes unidades conceptuales:

- la teoría general de sistemas (TGS),

- el concepto de interconexión de sistemas abiertos (OSI-ISO),

- las ciencias de la computación y las redes de computadoras,

- las sociedades de la información, los conocimientos y el aprendizaje,

- la virtualización,

- los avances en la 4⿳a revolución industrial, $\mathrm{y}$

- la metáfora ecosistémica como categoría organizativa.

Por la naturaleza dinámica del modelo educativo, el marco propuesto se diseñó para ser flexible con el propósito de que sea independiente de las funcionalidades $y$ características de la gran variedad de tecnologías y de marcas específicas que continuamente están evolucionado, apareciendo y desapareciendo, de tal manera que pueda ser aplicado en futuros rediseños.

\section{Análisis y propuesta de principios de diseño}

Para la definición del marco conceptual, se enfrentaron dos tipos de retos conceptuales para el diseño de los espacios virtuales:

a) el de su modelación y su organización

b) el del soporte socio-tecnológico para su implantación

\footnotetext{
2 Proyecto Seminario Educación Mediada por Tecnologías, cuyo objetivo fue el de articular teorías, enfoques, lenguajes, y procedimientos desde la perspectiva de cinco dimensiones de mediación técnico-pedagógica, en las etapas de planeación, puesta en marcha y evaluación del acto educativo. Las dimensiones son las siguientes: epistemológica; psicopedagógica; diseño y comunicación visual; científica y tecnológica; administrativa y vivencial. (https://sites.google.com/site/seminariovisiones/).
} 
Cada reto está organizado en unidades de análisis que están asociadas a teorías, conceptos y principios tecnológicos y sociales. A partir de cada una de ellas se derivaron los principios básicos específicos para el rediseño de un modelo educativo ecosistémico. El análisis propuesto, que se presenta en el diagrama de la figura 3, que va de lo general a lo particular, será la base de la propuesta del marco de referencia conceptual que se desglosan en los siguientes apartados.

Figura 3. Marco conceptual para el diseño de ecosistemas educativos

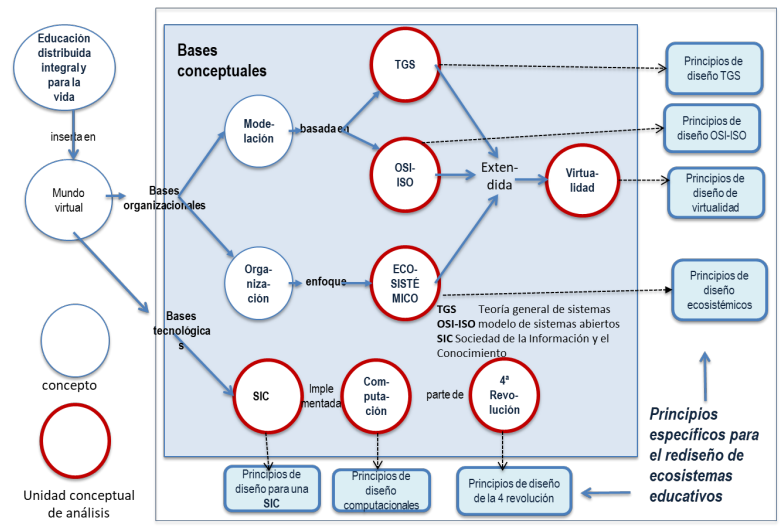

Fuente: elaboración propia, 2019.

\subsection{Reto organizativo}

¿Cómo fundamentar, modelar y diseñar espacios socio-virtuales flexibles y adaptables conformados por redes de sistemas abiertos autónomos e interdependientes a escala mundial que no pueden ser regidos por un control central y planeado?

Para dar respuesta a esta pregunta, se plantearon las siguientes unidades conceptúales de análisis:

a) la Teoría General de Sistemas (TGS) como la base conceptual de los ecosistemas virtuales,

b) concepto de Interconexión de Sistemas Abiertos (OSI por sus siglas en inglés, Open System Interconection) para el diseño de la interconexión de ecosistemas abiertos.

c) la metáfora ecosistémica como categoría organizativa para la coordinación de una red de ecosistemas educativos autónomos e interdependientes que están adaptándose, creándose y desapareciendo continuamente, $\mathrm{y}$ d) la virtualización para la extensión y ampliación a nivel mundial de los ecosistemas educativos

Para este reto, se presentan en este orden las unidades de análisis y por cada una de ellas se genera principios de diseño ecositémicos educativos.

\subsubsection{Teoría General de Sistemas (TGS)}

La teoría general de sistemas (TGS) surgió dese los años 40's y fue presentada por el biólogo Ludwig von Bertalanffy en 1969 (Bertalanffy, 1976). Aunque su origen fue ofrecer una concepción organicista de la biología, muy pronto se transformó en un marco teórico-práctico interdisciplinario fundamental e indispensable para poder comprender fenómenos sociales y naturales dinámicos y complejos modelados como una red de sistemas abiertos retroalimentados y autónomos que interactúan entre si en un ambiente determinado. Los sistemas abiertos se caracterizan por: su propósito-objetivo, totalidad, entropía, homeóstasis, sinergia, retroalimentación, modelo de entrada-transformación-salida y equifinalidad. La TGS es una herramienta teórica que no solamente permite la comprensión de fenómenos complejos sino también el diseño de redes de sistemas abiertos.

\subsubsection{Principios de diseño sistémicos}

Los sistemas educativos institucionales formales e informales tradicionales generalmente están diseñados como sistemas aislados, planeados y centralizados en un contexto generalmente sociocultural local específico y con un cierto grado de apertura al mundo. Aunque no son completamente rígidos, van adecuando sus programas a los cambios locales y globales que se van sucediendo y no siempre se dan al ritmo necesario que requieren las nuevas realidades locales y globales.

Desde la perspectiva de la TGS, un mundo educativo virtualizado a escala mundial puede modelarse y diseñarse como una gran red compleja y dinámica de sistemas computacionales abiertos que interactúan en el contexto de las sociedades de la información en un ambiente muy dinámico que está entre una gran entropía y su homeostasis, los sistemas que no logran adaptarse, desaparecen, y los que perduran se mantienen gracias a la 
retroalimentación interna y externa. Los sistemas educativos tienen sus propios propósitos y se van adaptando por la retroalimentación producida por los cambios internos de sus propios programas y procesos de formación y a los externos, que se derivan por la interacción con otros sistemas, tales como el laboral, el económico, social, el cultural etc.; lo que puede permitirles llegar a sus objetivos marcados en sus programas en condiciones diferentes a las previstas inicialmente o de lo contrario, desaparecen por falta de actualización. Desde esta perspectiva, el diseño de una educación inserta en el mundo no puede abstraerse de los otros sistemas ya que intervienen de manera dinámica y compleja otras variables, que van más allá de lo educativo-tecnológico, como son las sociales, culturales, económicas, políticas, etc. tanto a nivel local como global.

Dado este ambiente dinámico, es de suma importancia considerar todas las propiedades de la TGS y en particular las interacciones que existen entre los sistemas y sus bucles de retroalimentación que son las bases para su reconfiguración para que los sistemas educativos puedan evolucionar a la par con los cambios sociales y tecnológicos que continuamente se están dando en la actualidad.

En cuanto al rol del humano, éste es parte de todos los sistemas sociales a los que pertenece; de tal manera que un estudiante es a la vez un ciudadano, y puede ser también un trabajador y padre de familia; lo que le permite interactuar entre todos estos sistemas con los que se encuentra ligado y por lo tanto, con este enfoque, la educación virtualizada puede concebirse como integral y para la vida.

\subsubsection{Modelo para la interconexión de sistemas abiertos (OSI)}

Una aplicación del enfoque sistémico se encuentra en la red Internet de sistemas computacionales abiertos, programables, autónomos, interdependientes y distribuidos en todo el mundo, su origen se sitúa en 1969. A partir de entonces ha habido un desarrollo vertiginoso con la creación de tecnologías, dispositivos y programas cada vez más poderosos y accesibles, y que pueden integrarse gracias a que fueron diseñados a partir de un modelo conceptual de referencia para la
Interconexión de Sistemas Abiertos (OSI) que fue estandarizado en 1994 por la Organización Internacional de Normas (ISO por sus siglas en inglés, International Organization for Standardization) (OSI-ISO, 1994). Esta conceptualización y estandarización fueron desarrolladas en 1980 por Zimmermann (1980) y Day (1983) y posteriormente adoptada colaborativamente por una comunidad científica a nivel internacional que propuso la creación de un modelo conceptual para la interconexión de sistemas abiertos independientemente de las tecnologías y de las marcas de computadoras. Básicamente este concepto define que todo sistema programado en cualquier lenguaje computacional y tecnología específica de cómputo, por naturaleza debe tener la capacidad de comunicarse con cualquier otro sistema mediante los estándares internacionales establecidos para los protocolos de comunicación; en otras palabras, se definió, por consenso internacional, un lenguaje conceptual universal de comunicación que es adoptado por todas las nuevas marcas y tecnologías que vayan surgiendo. Este concepto es el que ha permitido que la red internet subsista a pesar de todos los cambios tecnológicos que van surgiendo $\mathrm{y}$ desplazando los que ya son obsoletos.

El gran aporte de este concepto es la red que permite plantear coordinaciones distribuidas, no necesariamente centralizadas, como las que fueron aplicadas en el mismo diseño de internet en los protocolos de enrutamiento de los mensajes en el que no existe un sistema centralizado que determine la ruta de un mensaje desde su origen hasta el punto final; el control está distribuido en los nodos ruteadores que con conocimientos parciales van buscando los caminos posibles hasta que al final un mensaje llega a su destino. Sin embargo, a pesar de esta experiencia, esta técnica de control distribuido no siempre es utilizada a nivel de las aplicaciones. En general la red está conformada por un conjunto de sistemas centralizados que dan servicios a sus usuarios, esta arquitectura centralizada se denomina cliente-servidor. Muy pocos sistemas computacionales son diseñados como redes distribuidas con un control descentralizado, dado que el centralizado es mas fácil de implementar. 
3.1.2.1. Principios de diseño para la conformación de redes de ecosistemas educativos abiertos

Los sistemas educativos virtualizados, aunque están insertos en la red Internet, en su diseño y operación siguen en general una organización planeada y centralizada, aún hay muy pocas iniciativas de redes educativas descentralizadas, aunque es la tendencia ${ }^{3}$.

Para contar con todo el potencial que ofrece una educación a escala mundial, ésta tendría que diseñarse como una red de sistemas educativos abiertos autónomos organizadas con técnicas de control distribuido que permitan encontrar sus equilibrios mediante una coordinación distribuida utilizando protocolos abiertos de negociación, inspirados, por ejemplo, en iniciativas tales como el de la Foundation for Intelligent Physical Agents de la IEEE Computer Society standards organization (FIPA, 2005), organismo que fomenta el desarrollo $y$ establecimiento de estándares de software para agentes heterogéneos que colaboran para resolver problemas complejos.

Fundamentalmente es necesario cambiar el enfoque de diseño sistemas educativos planeados y centralizados conectados a la red, al de un modelo conformado por una red de sistemas educativos abiertos, colaborativos, autónomos, interdependientes y adaptativos, utilizando técnicas de control distribuido. El potencial del control distribuido está aún en fase de investigación, pero se debe considerar pues es fundamental para encontrar equilibrios dinámicos en las redes de ecosistemas educativos.

\subsubsection{La metáfora ecosistémica}

Desde la perspectiva de la TGS y el concepto OSI es posible la modelación de redes dinámicas y

\footnotetext{
3 El concepto de redes educativas no es nuevo, entre otras iniciativas, se pueden encontrar la red Espacio Común de Educación de Superior a Distancia (ECOESAD), un consorcio nacional de universidades virtuales de México creado en 2008 (ECOESAD, 2008) que surgió del Espacio Común de Educación (ECOES), una red no virtual de instituciones educativas que promueven la movilidad física de estudiantes y profesores que se inspiró de una iniciativa europea. El ECOESAD permite compartir programas y recursos educativos, sin embargo, no aprovecha todo el potencial que puede ofrecer la distribución a otros niveles, como por ejemplo el de programas transversales con libre circulación virtual de diversas comunidades estudiantes organizadas en redes sociales.
}

complejas de grandes dimensiones, tal como lo es la red Internet. Para el diseño de redes dinámicas y evolutivas su gestión distribuida nos podría llevar al caos si no se define un paradigma de ordenamiento diferente al de los sistemas centralizados. En estas condiciones, es necesario una categoría organizativa nueva que pueda mantener equilibrios en un ambiente altamente dinámico que en ciertas circunstancias necesita restructurarse para adaptarse y evolucionar.

Con este enfoque, un ecosistema educativo se organizaría como un conjunto de estudiantes, profesores, gestores y agentes computacionales (seres bióticos) autónomos e interdependientes que ante circunstancias inesperadas encontrarían sus equilibrios en el aprendizaje, la producción y uso de conocimiento, recursos y servicios educativos abiertos (abióticos) mediante la colaboración y la negociación usando mecanismos de control basados en la competencia, la autorregulación y la autoorganización. Estos mecanismos son fundamentales para la adaptación y la evolución de los ecosistemas. Existen propuestas que no explicitan estos mecanismos, pero si los utilizan para plantear nuevos modelos educativos como, por ejemplo, entre otros tantos, el del "Aprendizaje Basado en el Pensamiento Cómo desarrollar en los alumnos las competencias del siglo XXI" de Swartz et al. (2007), miembro del comité de la International Conference on Thinking, ICOT, que propone una educación basada en habilidades como el pensamiento independiente, la capacidad comunicativa, la escucha activa, la empatía, la metacognición y la recopilación de datos a través de los sentidos; es decir, como un ecosistema de estudiantes y profesores autónomos que colaboran y se autorregulan en un proceso de enseñanza aprendizaje.

Es importante destacar que el enfoque ecosistémico, que incluye lo ecológico, no es nuevo; es muy común escucharlo en diferentes ámbitos no necesariamente con enfoque educativo. Por ejemplo, la ecología cognitiva de Levy (1998) que la considera como el espacio donde se definen y redefinen las posibilidades cognitivas individuales, institucionales $y$ técnicas; y que se inspira en el concepto de las 
ideas de Gregory Bateson (1972) sobre la ecología de la mente que la plantea a partir de varias disciplinas (biología, psiquiatría, antropología) y la considera como un conjunto de organismos vivos que nacen, crecen, se reproducen, se enferman, perviven, mueren, se adaptan, aprenden; o la concepción de las tres ecologías de Guattari (1989) que integra la ecología individual subjetiva, la social y la ambiental; o la ecología de medios propuesto por McLuhan (2011) que considera que la comunicación influye en el progreso social y que estos medios se generan y evolucionan como las especies biológicas; o los ecosistemas tecnológicos que han permitido un desarrollo sin precedentes mediante espacios reales y virtuales donde residen sistemas y productos abiertos que son desarrollados de manera autónoma por usuarios, organizaciones y empresas distribuidas en todo el mundo y que continuamente se están adaptando a las necesidades del mercado y de los usuarios 4 . Pero Igualmente, ya empieza aplicarse esta metáfora en la educación particularmente en el aprendizaje, por citar un ejemplo tenemos la ponencia sobre "Ecosistemas de aprendizaje y tecnologías sociales" de Freire (2011) quien planteó los problemas de la universidad tradicional para considerar otras formas de aprendizaje basadas en la tecnología; o, como Cristóbal Cobo (2010) que plantea que las instituciones nos preparan para la vida presente, pero no para el futuro; o, el de (Sánchez 2017) que presenta un programa y plataforma de autoformación interdisciplinaria e interinstitucional, sobre la educación mediada por TIC a partir de diferentes visiones disciplinarias, la psicopedagógica, tecnológica, epistemológica, de comunicación, vivencial; o los desafíos para replantear y organizar las instituciones de educación superior para esta era digital que está marcada por la desconfianza y la incertidumbre que se plantea en Ecologías de aprendizaje en la era digital: desafíos de la educación superior (González-Sanmamed et al. 2018). La tendencia actual se enfoca principalmente en el proceso de aprendizaje

\footnotetext{
${ }^{4}$ Entre las principales empresas que usan este enfoque están, Google, Apple y Microsoft, compañías económicamente muy poderosas que desarrollan sus sistemas operativos, aplicaciones y dispositivos móviles; los cuales a su vez hacen alianzas con otras empresas para extender sus productos.
}

definido como un espacio que permite integrar a personas, contenidos, servicios, normas, tecnologías, espacios y cualquier otro recurso con el propósito de mejorar este proceso. El enfoque propuesto en este trabajo, es diferente, es mas amplio, pues se considera la educación como una red de ecosistemas interdependientes a escala mundial en el que integra de manera distribuida los procesos de gestión y de enseñanza-aprendizaje, e igualmente toda la infraestructura tecnológica y administrativa.

En cuanto a propuestas propiamente ecosistémicas, está la iniciativa interdisciplinaria e internacional de Global Education Futures Report: educational ecosystems for societal transformation (Luksha et al, 2017) que parte de lo humano y de una educación como medio de transformación social mundial, su propuesta es el resultado de un análisis crítico del estado actual de la globalización de los mercados, en particular en lo que concierne a la crisis ambiental, y de la consideración de las tendencias que apuntan a una socialización global; a una conciencia ecológica; y a la necesidad de integrar conocimientos más allá de los racionales como lo son los producidos por las emociones, la creatividad, la espiritualidad y la sabiduría multicultural. Sin embargo, la propuesta puede verse como un catálogo de buenas intenciones pues carece de una fundamentación epistemológica con sentido crítico socialeconómico-político para poder ser aplicada en otros contextos, en particular a las realidades de países con culturas que se ven excluidas por una epistemología que privilegia el conocimiento científico; por ejemplo, la Ecología de Saberes de Boaventura de Sousa Santos (De Sousa, 2009) presenta un nuevo modelo educativo mundial intercultural, radicalmente distinto al actual que está dominado por las reglas del mercado del mundo neoliberal en el que vivimos. Este autor propone una ecología de saberes que incluye además del conocimiento científico, los saberes de otras culturas del mundo.

\subsubsection{Principios de diseño ecosistémicos}

Generalmente los sistemas educativos se diseñan desde un enfoque centralizado en la institución, en los planes y programas y recursos educativos $y$ finalmente en el profesor; y cuando se 
virtualiza se centra en una plataforma específica y en el uso de los servicios y recursos que ofrece la WEB. Estos sistemas se insertan de manera aislada en el mundo virtual para formar desde su modelo. Otro diseño sería concebirlos como ecosistemas educativos insertos en el mundo conviviendo y colaborando con todo los otros sistemas sociales y educativos, contando, además, con una flexibilidad que les permita su adaptación y evolución social-tecnológica, incluso en tiempo real, mediante los mecanismos de autorregulación y autoorganización. Y en cuanto a los procesos de enseñanza-aprendizaje, éstos se tendrán que diseñar, ya no basado en la relación centralizada profesor-estudiantes, sino en la colaboración entre actores autónomos e interdependientes que construyen conocimiento buscando sus propios equilibrios de enseñanzaaprendizaje de acuerdo con sus capacidades y necesidades, integrando comunidades internas y externas de aprendizaje y práctica, formándose (navegando) en los diferentes espacios educativo y sociales que ofrece la red.

\subsubsection{La virtualidad mediada por TIC}

En la actualidad, gracias a la red mundial Internet-Web se cuenta con cantidades enormes de dispositivos, conocimiento, usuarios, aplicaciones desarrolladas independientemente; con esta sistematización del mundo se dio un salto cuantitativo y posteriormente uno cualitativo. El mundo tiene otra extensión virtualizada y cualitativamente, ha replanteado prácticamente todo el quehacer humano. Cómo entender este fenómeno social-tecnológico desprendiéndonos de las funcionalidades deslumbrantes de todos estos dispositivos tecnológicos, ¿se puede comprender la tecnología sin tener en cuenta las consideraciones culturales? Es importante la pertinencia de esta última pregunta, pues existen corrientes con una visión puramente tecnológica que no consideran su relación con la cultura y su impacto social en el contexto histórico, social y económico en el que se vive.

Pierre Levy afirma, en su reporte al consejo europeo sobre el proyecto de nuevas tecnologías, que hay una relación íntima entre cultura y tecnología a partir de dos conceptos, el de cibercultura y ciberespacio.
Como empleo a menudo los términos «ciberespacio» y «cibercultura». parece útil proporcionar desde ahora mismo una breve definición de ellos. El ciberespacio (que llamaremos también la «red») es el nuevo medio de comunicación que emerge de la interconexión mundial de los ordenadores. El término designa no solamente la infraestructura material de la comunicación numérica, sino también el oceánico universo de informaciones que contiene, así como los seres humanos que navegan por él y lo alimentan. En cuanto al neologismo «cibercultura», designa aquí el conjunto de las técnicas (materiales e intelectuales), de las prácticas, de las actitudes. De los modos de pensamiento y de los valores que se desarrollan conjuntamente en el crecimiento del ciberespacío. (Levy, 1997, p1).

A partir de esta perspectiva, el autor extiende el concepto de inteligencia humana a una colectiva en la que integra a la artificial; incluso propone la creación de una nueva antropología para comprender este nuevo fenómeno cultural (Levy, 2004). Sin embargo, cabe preguntarse, ¿de dónde reside el potencial de la virtualización?; la respuesta se encuentra en el mismo autor, (Levy, 1999), la define como una fuerza que existe en potencia, pero no en acto y no se opone a lo real sino a lo actual donde la virtualidad y la actualidad sólo son dos maneras de ser diferentes, Y cuando se actualiza (se implementa) se convierte en una nueva realidad. Se trata de un concepto amplio que se aplica a cualquier tecnología, por ejemplo, el libro que extiende la comunicación oral, y que con la digitalización y las redes, lo extiende a una a escala mundial sin precedentes. Desde esta perspectiva, la virtualización de una actividad humana se realiza en un espacio con características diferentes a las reales, por ejemplo, una educación virtualizada mediante un sistema de gestión del aprendizaje (LMS por sus siglas en inglés) funciona con interacciones diferentes a las de una educación presencial centrada en un espacio real, el aula.

Levy no va más allá de estas conceptualizaciones pero en este trabajo, se profundizó en el potencial de la virtualidad mediante las TIC al caracterizarla de la manera siguiente: en el mundo virtual, gracias a la propiedad fundamental la deslocalización geográfica, todo se virtualiza, subespacios, procesos, contenidos digitales y actores (sujetos, objetos, procesos y subespacios); todo espacio es 
abierto y sus contenidos se acceden (se navegan) en tiempo real simplemente nombrándolos o señalándolos. Además en estos espacios, no solo se almacena datos, sino también se pueden memorizar la historia (trayectorias) de sus accesos a objetos, sujetos y otros espacios, que mediante algoritmos basados inteligencia artificial permiten analizar comportamientos y predecirlos incluso en tiempo real. Finalmente, gracias al potencial de la programación y auto programación, estos espacios se pueden reconfigurar incluso automáticamente; esta propiedad es la más poderosa, pues a través de ella se pueden implementar espacios flexibles y adaptativos, incluso en tiempo real, necesarios para convivir en la red virtual compleja y evolutiva de las sociedades de la información y los conocimientos conformadas por espacios virtuales autónomos e interdependientes.

Resumiendo, la virtualidad se puede definir como una extensión espacial de la realidad conformada por una red de subespacios deslocalizados abiertos, autónomos e interdependientes, sustentados en el poder de las redes de computadoras, contando todos con las siguientes propiedades:

- Independencia de un lugar geográfico (deslocalización) de los espacios y sus contenidos accesibles (navegables) en tiempo real con solo nombrarlos o tocarlos.

- Espacios abiertos conteniendo objetos, sujetos, procesos y subespacios virtualizados, que pueden pertenecer a otros espacios, y que pueden desplazarse entre los espacios de manera inmediata.

- Libre circulación de espacios y sus contenidos en la red

- Todo espacio tiene memoria

- Todo espacio puede programarse y reconfigurarse lo que facilita adaptarse a eventos internos o externos, mediante mecanismos de autorregulación y autogestión

Gracias a estas características, la creación de espacios virtuales ecosistémicos es mucho más fácil implementarla que los reales, naturales y sociales, pues éstos están supeditados a los límites de los espacios físicos geográficamente localizados; mientras que la virtualización, al caracterizarse precisamente por su deslocalización geográfica, permite la programación, la reprogramación y la auto programación de espacios abiertos, flexibles y navegables en tiempo real, por ejemplo, navegando de un espacio educativo formal a uno informal de algún chat o a otro de alguna red social. Una prueba de este gran potencial es su aplicación en la virtualización del mundo, las sociedades de la información, los conocimientos y el aprendizaje que nos permite navegar libremente en todos los sistemas que residen en ese mundo virtualizado.

3.1.4.1. Principios de diseño para una formación desde la virtualización

Los sistemas educativos virtualizados han permitido a las instituciones extender la educación de las instituciones mas allá de sus fronteras, y por ser virtual no significa que no sea real, como lo expresa el Grupo de Investigación UBUNTU en el "Estado del arte sobre la articulación de modelos enfoques y sistemas en educación virtual" (Cárdenas et al. 2017), "Como conclusión en este apartado, si se utiliza el término educación virtual, se hace referencia a aquella educación a la cual simplemente le falta laæsं exi, poqealer ital a d, sá

idéntica a la real" (p. 23). Sin embargo, generalmente los sistemas educativos formales virtualizados en el mundo, se diseñan como sistemas centralizados y no aprovechan en sus programas de formación todos los otros sistemas educativos y sociales que están insertos en el mundo virtual. Pero si se piensa en una educación distribuida, integral y para la vida a escala mundial basada en una red de ecosistemas educativos autónomos y colaborativos, el alcance de la educación se ampliaría significativamente pues pasaría ser parte de la cibercultura. Desde esta perspectiva, la educación puede aspirar no solamente a conformar una sociedad de los conocimientos, si no, de la sabiduría basada en nuevo tipo de inteligencia, la colectiva que supera a la individual (Levy, 2004). Es importante destacar que en esta espacio culturaltecnológico se integra un nuevo tipo de actor, los agentes computacionales, basados en inteligencia artificial (IA), que se pueden programar y reprogramar como entes autónomos y proactivos que conviven en el 
mismo ecosistema. En este dominio ya existen aplicaciones disponibles como por ejemplos los chatboot $^{5}$ que son sistemas que pueden apoyar a los procesos de enseñanza-aprendizaje mediante algoritmos que van aprendiendo de las interacciones con los humanos. Para el diseño de espacios educativos virtuales es importante integrar a estos nuevos actores que pueden colaborar en la autoorganización y autorregulación en los procesos de gestión y de enseñanza-aprendizaje. Y por supuesto, integrar los principios de cibercultura e inteligencia colectiva.

\subsection{Reto del soporte tecnológico}

Generalmente en la educación virtualizada, las TIC se consideran sólo como recursos digitales, sin embargo, estas tecnologías han evolucionado y se han convertido en sistemas complejos, como por ejemplo, la red Internet-WEB que tiene su propia dinámica y está en continua evolución y que ofrece una cantidad ilimitada de recursos; por lo que es necesario un nuevo enfoque más allá de considerarlas simplemente como recursos. Para aprovechar mejor este soporte tecnológico, a partir de un enfoque crítico, se plantearon las siguientes unidades de análisis:

a) el entorno educativo-tecnológico que representa en su conjunto el mundo virtual de las sociedades de la información y los conocimientos

b) el gran potencial de las redes de computadoras y de las ciencias de la computación

c) la integración de nuevas tecnologías que han dado origen a la $4^{\underline{a}}$ revolución industrial que se derivó de una fusión de tecnologías digitales, biotecnología, internet de las cosas, inteligencia artificial, robótica, genética, computación cuántica, etc.

A partir de estas unidades de análisis se plantean, para cada una de ellas, principios de diseño específicos.

\footnotetext{
${ }^{5}$ Chatboot es un programa basado en inteligencia artificial que simula una conversación con el usuario. En la educación se usan como asistentes para resolver dudas sobre los procesos de enseñanza.
}

\subsubsection{Sociedades de la información y los conocimientos}

La premisa de este trabajo parte de que el mundo virtual es el gran entorno educativo. Este espacio, denominado, las sociedades de la información y los conocimientos, fue definido formalmente por la UNESCO (2005) donde se adopta como estrategia educativa mundial el concepto de sociedades del aprendizaje. Dicho concepto ya había sido planteado en 1968 por Hutchins (1968) quien considera que los conocimientos no están contenidos sólo en las instituciones educativas y que además no se limitan a la formación parcial sino a la integral, conjuntando educación, formal, no formal e informal. Todo este conocimiento, que no deja de crecer, se encuentra inserto en este mundo virtual que está conformado por dos grandes espacios virtuales interdependientes entre sí; el primero, donde conviven una gran diversidad de sistemas socio tecnológicos educativos, culturales, sociales, etc.; y el segundo, por el entorno tecnológico conformado por una gran diversidad de aplicaciones, recursos, servicios digitales, información, conocimientos, experiencias, etc. almacenados en el BIGDATA y en la NUBE; recursos digitales abiertos que son generados y consumidos por los sistemas sociotecnológicos del primer espacio.

3.2.1.1. Principios de diseño para insertar la educación a las sociedades de la información y los conocimientos

Como parte del espacio social-tecnológico, generalmente los sistemas educativos están concebidos como sistemas centralizados y no colaborativos con otros sistemas. Es importante dejar de diseñarlos como sistemas centralizados a los que básicamente se conectan estudiantes y profesores de la institución y utiliza sólo la WEB como un gran repositorio de recursos educativos que complementan los recursos educativos de los programas de la institución; cuando en realidad las bases de conocimiento creadas en la red los superan muy ampliamente; el conocimiento local generado por una institución contribuye al conocimiento mundial generado por otras, por lo que es mucho más amplio y puede ser compartido. Además, es importante 
considerar que este entorno tecnológico no es sólo un conjunto de recursos abiertos; es igualmente una red de sistemas sociotecnológicos complejos que tienen su propia dinámica y que están constantemente incidiendo en los sistemas educativos. Por ejemplo, integrar a un programa educativo una red social simplemente como un recurso más, es una simplificación que no permite aprovechar todo el potencial comunicativo de las redes sociales. Un buen ejemplo del aprovechamiento de un entorno tecnológico es "La escuela gratuita 42" diseñada para programadores y desarrolladores de software en París, Francia, (École 42, 2013). Este modelo se basa en un espacio que permite a sus estudiantes el uso de recursos y experiencias desarrolladas por otros desarrolladores y es guiada por proyectos que no requieren del seguimiento de profesores. Esta experiencia podría ser mejor aprovechada si se integrara en una red como un ecosistema educativo más para una formación integral y para vida.

\subsubsection{Redes de computadoras y las ciencias de la computación}

Más allá de los dispositivos y funcionalidades de las redes de computadoras su gran potencial está en sus fundamentos, las ciencias formales de la computación (Denning et al. 1989) que tienen su origen moderno a principios de 1940 y son el resultado de la confluencia de la teoría de algoritmos, lógica matemática y la invención del programa almacenado en una computadora electrónica y que posteriormente dio origen a la 3 a revolución industrial, la era digital, originada por la red mundial Internet y antecesora de la $4^{\mathrm{a}}$ que se está desarrollando. La gran capacidad de una computadora es su capacidad de memorizar y procesar algoritmos y que aunado a las redes, estas capacidades se amplían muy significativamente y además, agrega una nueva capacidad, la de comunicación digital y finalmente, la de poder distribuir el cómputo que permite resolver problemas complejos al descomponerlo en módulos programables en lugar de implementarlo con un solo programa centralizado. Es este principio que dio origen a las redes Internet-WEB.
El potencial de las redes de computadoras se puede resumir a partir de las siguientes capacidades:

- La de memorización distribuida en las redes de computadoras, la base virtual y mundial de datos, conocida como el BIGDATA ${ }^{6}$ que representa el acervo vivo, abierto y creciente de información y los conocimientos creado socialmente como nunca se había logrado en la humanidad.

- La de procesamiento distribuido en red que mediante aplicaciones y sistemas desarrollados por usuarios y empresas independientes, los sistemas y aplicaciones Web almacenadas en la NUBE

- , representan el repositorio de aplicaciones y sistemas más grande del mundo creado colectivamente.

- La de ser máquinas reprogramables por algoritmos, una computadora puedes ser desde un procesador de textos hasta un simulador profesional de vuelo. E incluso puede reconfigurarse en tiempo real para cambiar su algoritmo original, que en inteligencia artificial se le llama autoaprendizaje. Esta propiedad es esencial para la adaptación.

- La de interconexión que permite una interacción ágil entre usuarios y organizaciones; las redes sociales son el ejemplo más importante de este potencial.

- El acceso a todo lo conectado en la red en tiempo real, sin importar distancias, gracias a la gran velocidad de las telecomunicaciones.

3.2.2.1. Principios de diseño desde las redes y ciencias de la computación

Para el diseño de sistemas educativos virtualizados con enfoque ecosistémico se debe aprovechar todo el potencial que ofrecen las redes de computadoras y las ciencias de la computación:

a) su gran capacidad de memorizar que con las redes se ha llegado crear el mayor

\footnotetext{
${ }^{6}$ En el BIGDATA, que no deja de crecer, se producen millones de datos por segundo, por lo que es casi imposible saber con exactitud cuánta información contiene, se calcula en una decena de ZETABYTES (cifra de un 1 seguido de 27 0's) y se dice que hay el equivalente de centenas de billones de libros, una cantidad creciente e inconmensurable.
} 
espacio virtual, el BIGDATA y la NUBE, y aprovechar todos los recursos abiertos de información, servicios y aplicaciones almacenados en estos espacios

b) diseñar aplicaciones complejas como una red distribuida de aplicaciones básicas, no diseñarlas como sistemas centralizados monolíticos

c) aprovechar la capacidad de programación y reprogramación, lo que permite su autorregulación y autoorganización incluso en tiempo real, y

d) aprovechar todos los medios de interacción hipermedia que ofrece la red para interactuar, comunicar y negociar con todos los otros sistemas con los que se convive.

\subsubsection{Revolución industrial y revolución cultural}

La 4a a revolución industrial se derivó de una fusión de tecnologías digitales, biotecnología, internet de las cosas, inteligencia artificial, robótica, genética, computación cuántica, etc., que Klaus Schwab, fundador y presidente ejecutivo del Foro Económico Mundial, la definió en su 4o foro como la Industria 4.0 (World Ecomic Forum, 2016). Estos nuevos avances están nuevamente revolucionado prácticamente toda la actividad humana, y por ende a la educación; Esta nueva revolución está replanteando las categorías del aprendizaje en red que se desarrolló en la anterior, la digital; ahora es necesario, como lo plantea Escudero (2018), tomar como punto de partida la ontología poshumanista y la epistemología de la teoría del actor-red. Sin embargo, es importante destacar que las tecnologías son productos humanos, tal como lo expresa en Técnica y Autonomía. Reflexiones teórico-filosóficas Valle (2019) "Todo instrumento es humano y todo lo humano es instrumento. Toda educación es técnica en tanto es un medio y un hacer humano; toda técnica es educativa porque es un medio para lograr algo, y es un hacer que forma al ser humano" (p. 64), sin olvidar que su uso conlleva un impacto social y ambiental.

Es importante destacar, que este enfoque ecosistémico no solamente es una tendencia, sino, también una necesidad para comprender y diseñar sistemas complejos, y necesarios para resolver los grandes problemas que este mundo interconectado nos está continuamente planteando. Esta tendencia está dando origen a teorías, conceptos, metodologías que permitirán no solo comprender mejor el planeta, sino, poder transformarlo desde una postura humana y comprometida con los saberes multiculturales y una nueva convivencia con la naturaleza, tal como lo expresa Pablo González Casanova:

\begin{abstract}
La Revolución Científica de nuestro tiempo ha sido equiparada a la que ocurrió en tiempos de Newton. Hoy ya no podemos pensar sobre la naturaleza, la vida y la humanidad, sin tomar en cuenta los descubrimientos que se iniciaron con la cibernética, la epistemología, la genética, la computación, los sistemas autorregulados, adaptativos y autopoiéticos, las ciencias de la comunicación, las ciencias de la organización, las del caos determinista, los atractores y los fractales. La profundidad de esos descubrimientos va más allá de sus claras manifestaciones científicas y técnicas. Incluye nuevas formas de pensar y actuar que comprenden las llamadas ciencias de la complejidad y las tecnociencias. (Gonzáles Casanova 2017, p13).
\end{abstract}

El gran poder de estas nuevas ciencias y tecnologías, por sí solas, no cambian las estructuras que socialmente crean desigualdades; es necesario darles un sentido crítico y humano como lo plantea el autor. Por ejemplo el BIGDATA, como lo considera O'Neil (2017) en Armas de destrucción matemática: como el BIGDATA aumenta la desigualdad y amenaza la democracia, porque las decisiones son tomadas ahora por algoritmos inteligentes para favorecer los intereses comerciales de una minoría; pero al mismo tiempo, como lo expone Rendueles (2016), la ciudadanía digital plantea la posibilidad de una alternativa vigorizante al proceso de desafección política característico de las sociedades de masas ultraconsumistas.

3.2.3.1. Principios de diseño de una formación virtualizada a partir de las tecnociencias

El diseño de un ecosistema educativo inserto en el mundo virtual tiene que considerar las ciencias de la complejidad, las tecnociencias, los ecosistemas y con el potencial de la virtualidad, utilizar todos los recursos culturales y tecnológicos para una educación a escala planetaria de manera racional. Sin embargo, la 
tecnología y el conocimiento no son neutros, conllevan para bien y para mal un sentido humano por lo que es pertinente y fundamental explicitar el sentido que tendría la virtualización; en esta propuesta, es él de una educación como medio de transformación social en el contexto de un mundo virtualizado complejo conviviendo con el mundo natural que están en crisis.

Particularmente es importante considerar las tecnologías de la inteligencia artificial (IA), que están dotando de un soporte "inteligente" a todo tipo de actividad mediante un nuevo actor proactivo, ya sea un agente físico (como los robots) o algoritmos, que conviven con los actores humanos, profesores, estudiantes y administradores. La introducción de los agentes basados en IA está replanteando los procesos de enseñanza aprendizaje. En este sentido, es muy importante aprovechar el gran potencial que ofrece la IA para la educación en los procesos de gestión y de enseñanza-aprendizaje utilizando algoritmos inteligentes que aprendan de los actores humanos. Estas aplicaciones son un tema de investigación y desarrollo que hay que considerar en los diseños, pero, sin dejar de considerar críticamente el impacto social en su uso, ya que, tienden a desplazar actividades humanas que siguen siendo necesarias como la del profesor, desde luego con otro rol, el de apoyar los procesos de enseñanza-aprendizaje mediante el uso de agentes computacionales inteligentes y proactivos.

\section{Discusión y conclusiones}

Dada la complejidad que implicaría la implementación de una formación virtual, distribuida, integral y para la vida a escala mundial desde un enfoque ecosistémico, se hace indispensable la creación de un marco teóricoconceptual para fundamentar rediseños flexibles de este nuevo paradigma de modelo educativo propuesto. A pesar de que existen teorías, conceptos y proyectos con un enfoque ecosistémico, como fue analizado en el inciso 1.3.1 Metáfora ecosistémica, éstos son abordados desde diferentes ámbitos como el tecnológico, el de la comunicación, lo social, lo cultural, en el aprendizaje, etc., y no hay aún, uno que integre todas estas experiencias, teorías y conceptos educativos, sociales, culturales, filosóficos, epistemológicos y tecnológicos para una formación como la propuesta en este trabajo. En este sentido éste trabajo representa un primer acercamiento de conceptualizaciones sociotecnológicas enfocadas a la educación como un fenómeno social-cultural-epistemológico, presentado como un dialogo entre educación y tecnología muy necesario para fundamentar mejor futuros rediseños del nuevo paradigma de redes de ecosistemas educativos. De manera general, se propone fomentar un diálogo epistémico que debe ampliarse con la participación de todas las disciplinas que están involucradas en el modelo de formación propuesto. $\mathrm{Y}$ en este sentido, este marco representa un primer aporte que debe complementarse y ampliarse, siempre desde una perspectiva crítica con sentido social y ambiental.

Concretamente en este trabajo se propuso las bases de un marco teórico-conceptual del que se derivó en una herramienta conformada por un conjunto de principios flexibles para el rediseño de una educación basada en una red de ecosistemas educativos colaborativos $y$ adaptativos para una formación distribuida, integral y para la vida en el marco del mundo virtual de las sociedades de la información, los conocimientos y el aprendizaje. Donde la educación se le considera, tanto en su gestión administrativa como en la de los procesos de enseñanza aprendizaje, distribuida en una red colaborativa de ecosistemas abiertos que conviven con el mundo virtualizado y que encuentran sus equilibrios en su evolución mediante los mecanismos de autorregulación y autoorganización.

Desde luego, esta propuesta es organizacional y no profundiza sobre los procesos de gestión y de enseñanza-aprendizaje. El enfoque ecosistémico plantea grandes retos educativos, entre otros, por citar un ejemplo, su aplicación en el curriculum visto como un ecosistema abierto, flexible, distribuido, que integre educación formal, no formal e informal, el mundo del trabajo y el cultural; éste es un problema abierto aún en fase de investigación y desarrollo.

Concretamente, el resultado de este trabajo es una propuesta de principios flexibles, definidos a partir de un marco conceptual socio-tecnológico 
para el rediseño de modelos educativos con alcance mundial desde la perspectiva ecosistémica. En esta propuesta se destacan además los siguientes aportes:

a) La profundización del concepto de virtualización de Levy (1999) con la caracterización del potencial de la virtualidad desde la perspectiva de la cibercultura para el rediseño de ecosistemas educativos, adaptativos, proactivos, reconfigurables, navegables; características necesarias para la adaptación y evolución.

b) Además de aplicar el potencial del enfoque ecosistémico a nivel organizativo, se propone también el vivencial, donde se considera que todos los actores y sistemas son autónomos y colaborativos y que encuentran sus equilibrios en los diversos procesos de enseñanza aprendizaje distribuidos en todo el mundo mediante la colaboración y la negociación utilizando los mecanismos de regulación y autoorganización para adaptarse continuamente a las necesidades de formación que requieren el mundo real y virtual que están en continua evolución.

c) El impacto social de la tecnología; ésta no es neutra en cuanto a su diseño y uso, en la actualidad la gran tendencia es su comercialización en beneficio de unas cuantas empresas tecnológicas que finalmente han creado grandes desigualdades, no solamente como brechas tecnológicas ${ }^{7} \mathrm{y}$ de conocimiento, sino también, económicas las cuales han generado crisis sociales, económicas $\mathrm{y}$ políticas, provocado un impacto negativo en el medio ambiente del planeta. Por lo que se hace necesario plantear principios éticos de rediseño basados en una nueva relación con el medio ambiente, la solidaridad y el bienestar de todas las culturas del mundo, como el plantado en las epistemologías del sur (De Sousa 2009) y basados en nuevas formas de pensar y actuar como lo refiere González Casanova (2017).

d) El considerar la conciencia ambiental, que está marcando una preocupación y una tendencia a escala planetaria, que busca encontrar soluciones a los problemas complejos sociales, económicos, políticos y ambientales que el mundo está enfrentando, donde la educación como ecosistema y como medio de transformación social a nivel local y mundial tiene un rol muy importante.

Finalmente, con este trabajo se pretende contribuir en la fundamentación teórica y conceptual en el área general interdisciplinaria de la educación virtualizada, y en particular, en el área de nuevos paradigmas de formación como el aquí planteado, una formación distribuida, integral y para la vida a escala mundial basada en una red de ecosistemas educativos insertos en el mundo virtual de las sociedades de la información, los conocimientos y el aprendizaje.

\section{Agradecimientos}

Este trabajo ha sido el resultado de una investigación que se realizó en una estancia sabática en la Facultad de Educación de la Universidad de Málaga España, de marzo a agosto del 2018, financiada por el Programa de Apoyos para la Superación del Personal Académico de la UNAM (PASPA) 2018.

${ }^{7}$ Básicamente en el mundo occidental son Apple, Google, Microsoft, Amazon y Facebook que dominan el mercado tecnológico con un poder económico que trasciende a estados nacionales. En 20017, si Apple fuera un país, tendría un tamaño similar al de la economía turca, holandesa o suiza, https://elpais.com/economia/2017/11/03/actualidad/150971436 6_037336.html 


\section{Referencias}

Bertalanffy, von Ludwig (1976). Teoría general de los sistemas. Fundamentos, desarrollo, aplicaciones. México: Fondo de Cultura Económica.

Bateson, Gregory (1972). Steps to an Ecology of Mind: Collected Essays in Anthropology, Psychiatry, Evolution, and Epistemology. University Of Chicago Press.

Cárdenas, E. P. L., García, P. A. M., Piraguata, J. A. R., \& Padilla, A. S. (2017). Estado del arte sobre la articulación de modelos enfoques y sistemas en educación virtual. Escuela de Ciencias de la Educación ECEDU. Recuperado de http://hemeroteca.unad.edu.co/index.php/book/article/view/1969/2189

Cobo, Cristobal y Movarek Jhon. (2011). Aprendizaje Invisible, Hacia una nueva ecología de la educación. Libro electrónico Recuperado de http://www.razonypalabra.org.mx/varia/AprendizajeInvisible.pdf

Consorcio Universidad 2030 Virtual Educa (2019). Recuperado de https://virtualeduca.org/universidad2030/consorcio-universidad-2030

Day, John y Zimmermann, H. (1983). The OSI reference model. Proceedings of the IEEE, 71(12), 13341340.

Denning P.J. et al. (1989). Computing as a Discipline. Communications of the ACM. Association for Computing Machinery, 32(4), 9-23.

De Sousa, B. (2009). Introducción Epistemología del Sur. Libro en versión electrónica: http://www.boaventuradesousasantos.pt/media/INTRODUCCION_BSS.pdf

ECOESAD (2008). Espacio Común en Educación a Distancia. Sitio Recuperado de http://www.ecoesad.org.mx/

École 42. (2013). École 42. Recuperado de https://www.42.fr/

Escudero, A. (2018). Redefinición del "aprendizaje en red" en la cuarta revolución industrial. Apertura 10(1), 149-63. Recuperado de: http://www.scielo.org.mx/scielo.php?script=sci_arttext\&pid=S1665-61802018000100149

FIPA Foundation for Intelligent Physical Agents, (2005). IEEE Computer Society standards organization, Recuperado de http://www.fipa.org/

Freire, J. (2011). Ecosistemas de aprendizaje y tecnologías sociales, nómada, blog de juan freire. Recuperado de $\quad$ https://nomada.blogs.com/jfreire/2011/05/ecosistemas-de-aprendizaje-ytecnolog\%C3\%ADas-sociales-tedxuimp.html

González Casanova, P. (2017). Las nuevas ciencias y las humanidades: de la academia a la política. 1a. Ed. Ciudad Autónoma de Buenos Aires. CLACSO.

González-Sanmamed, M.; Sangrà, A.; Souto-Seijo, A. y Estévez, I. (2018). Ecologías de aprendizaje en la Era digital: desafíos para la educación superior. Publicaciones, 48(1). Facultad de Educación y Humanidades del Campus de Melilla, España. 25-45. Recuperado de: https://pdfs.semanticscholar.org/2ce0/26d7ee9dda43df39caec75a483dfecddfde5.pdf?_ga=2.859 2055.36999549.1581194882-665151805.1581194882

Guattari, P. F., Vázquez Pérez, J. y Larraceleta, U. (2000). Las tres ecologías. Valencia: Pre-Textos. Recuperado https://www.arteuna.com/talleres/lab/ediciones/FelixGuattariLastresecologas.pdf

Hutchins, R. (1968). The learning society. University of Chicago Press.

Levy, P. (1997). Cyberculture. Rapport au Conseil de l'Europe dans le cadre du projet Nouvelles technologie: coopération culturelle et communication. Vol. 1. Paris: Ed. Odile Jacob.

-(1998). Tecnologías de inteligencia: el futuro del pensamiento en la era de la informática. Editorial 34

-(1999). ¿Qué es lo virtual?. Barcelona: Paidós. Recuperado de http://www.hechohistorico.com.ar/Archivos/Taller/Levy\%20Pierre\%20\%20Que\%20Es\%20Lo\%20Virtual.PDF 
-(2004). Inteligencia colectiva. Por una antropología del ciberespacio. Biblioterca Virtual en Saude. Recuperado de http://inteligenciacolectiva.bvsalud.org/public/documents/pdf/es/inteligenciaColectiva.pdf

Luksha P., Cubista J., Laszlo A., Popovich M. y Ninenko I (Eds.). (2017). Global Education Futures Report: educational ecosystems for societal transformation. Recuperado de http://www.globaledufutures.org/images/people/GEF_april26-min.pdf

McLuhan, E. y McLuhan, M. (2011). Theories of communication. Nueva York: Peter Lang.

O'Neil, K. (2017). Armas de destrucción matematica. Como el big data aumenta la desigualdad y amenaza la democracia. Madrid: Capitan Swing libros.

OSI-ISO (1994), Modelo para la interconexión de sistemas abiertos. Recuperado dehttp://standards.iso.org/ittf/PubliclyAvailableStandards/s020269_ISO_IEC_74981_1994(E).zip.

Rendueles, C. (2016). La ciudadanía digital. ¿Ágora aumentada o individualismo postmaterialista. Revista Latinoamericana de Tecnología Educativa RELATEC 15(2). España, 15-24.

Sánchez, V. (2017). Cap. 16: Principios de un modelo de ecosistema de aprendizaje basado en las experiencias de un seminario. Aprendizaje creador y nuevas prácticas pedagógicas, Congreso Internacional de Transformación Educativa. Amapsi Editorial, México, 334-353.

Secretariat of the Convention on Biological Diversity (2004). Enfoque por ecosistemas. Montreal, QC, Canada: Secretaría del Convenio sobre la Diversidad Biológica. Recuperado de https://www.cbd.int/doc/publications/ea-text-en.pdf

Swartz, R. J., Reagan, R., Costa, A. L., Beyer, B. K., y Kallick, B. (2015). El aprendizaje basado en el pensamiento. Cómo desarrollar en los alumnos las competencias del siglo XXI. Ediciones SM España.

Valle, A. (2019). Técnica y Autonomía. Reflexiones téorico-filsóficas. Hernández, G., Ortiz, Henderson, Nájera, Gladys; Espinosa, Ozziel, (Eds). Futuros digitales exploraciones socioculturales de las tic.

Worl Ecomic Forum (2016). The Fourth Industrial Revolution: what it means, how to respond. Recuperado de https://www.weforum.org/agenda/2016/01/the-fourth-industrial-revolution-what-it-meansand-how-to-respond/.

Zimmerman, H. (1980). OSI Reference Model - The ISO Model of Architecture for Open Systems Interconnection. IEEE Transactions on Communications, 28(4), 425-432. 